\title{
Huffman and colleagues' response to Abramson and colleagues' article on statins in low risk people
}

\author{
Mark Huffman assistant professor of preventive medicine and medicine-cardiology ${ }^{1}$, Fiona Taylor \\ editor, Cochrane Heart Unit ${ }^{2}$, Shah Ebrahim professor of public health ${ }^{2}$
}

${ }^{1}$ Departments of Preventive Medicine and Medicine-Cardiology, Northwestern University Feinberg School of Medicine, Chicago, IL, USA; ${ }^{2}$ Department of Noncommunicable Disease Epidemiology, London School of Hygiene and Tropical Medicine, London, UK

The $B M J$ Analysis article on statins in low risk people stated, "the evidence does not show that the benefits of statins in low risk patients outweigh the harms and that the advice for treatment of this group should not be changed." "It criticises our Cochrane systematic review on statins for the primary prevention of cardiovascular disease for not coming to the same conclusion. ${ }^{2}$ Readers of the $B M J$ may be interested in our views on their arguments.

This article predated by three weeks the publication of the 2013 American College of Cardiology/American Heart Association (ACC/AHA) cholesterol treatment guidelines (12 November 2013), but statements were made about "proposed standards" without full knowledge of these guidelines. Notably, none of the authors were acknowledged reviewers of the ACC/AHA guidelines. ${ }^{3}$

Abramson and colleagues state: "Under the proposed 2013 standards, however, no level of risk would preclude statin therapy, raising the question whether all people over the age of 50 should be treated." Neither the Cochrane review nor the ACC/AHA cholesterol guidelines proposed treatment for everyone over the age of 50 years. The ACC/AHA guidelines recommend that initiation of moderate intensity statin treatment be considered for patients with a predicted 10 year "hard" risk of arteriosclerotic cardiovascular disease of $5.0 \%$ to less than 7.5\%. The Cochrane review questioned the feasibility and desirability of having to treat most people over the age of 50 years with a statin.

The authors comment that the inclusion of four additional trials "did not substantially alter the previously documented effect of statin therapy." The updating of the evidence base resulted in an expected narrowing of confidence intervals, and the addition of the JUPITER trial added important evidence on diabetes risk.

The authors consider that for statins to have a place in primary prevention in people in lower strata of cardiovascular disease risk these drugs should reduce total mortality, and they estimate a relative risk of 0.95 (95\% confidence interval 0.86 to 1.04 ). However, the authors included people with and without previous vascular disease in this estimate. We conducted the appropriate analysis using only low risk people $(<5 \% ; 5-10 \%)$ without previous vascular disease (table $\downarrow$ ). In the lowest risk category $(<5 \%)$, the number of total deaths was small ( $1 \%$ of control group participants dying over four years) and non-cardiovascular disease causes of death exceeded deaths from cardiovascular disease by more than 2:1. However, the risk of experiencing a major vascular event (fatal and non-fatal) was $0.6 \%$ per year, which was reduced by statins (relative risk $0.62,95 \%$ CI 0.47 to $4.81 ; 167$ events on statin $v 254$ events on control; similar effect size across major coronary event, stroke, and revascularisation) in this low risk group (fig 1 in Cholesterol Treatment Trialists' 2012 paper $^{4}$ ). No strong evidence of benefit for total mortality was seen because other causes of death make up a greater proportion of total deaths, and it is unlikely that taking statins influences these non-cardiovascular disease deaths.

We disagree with Abramson and colleagues' statement that the "best indication of the net effect of a treatment on overall health is the total number of serious adverse events-which include deaths from all causes, hospital admissions, prolongations of admission, cancer, or permanent disability." It is incorrect to give each of these events a similar weight. Deaths, disability, and prolongation of admission are quite different outcomes that would not be given similar weight by most patients. ${ }^{5}$

While criticising the randomised controlled trials, the authors use low quality evidence from observational studies to support their statements about the hazards associated with statins, even though the risk of bias is likely to be high in such studies. They also conflate muscle pain (myalgias), an important side effect of statins, with myopathy, a rare and more serious problem, both of which warrant ongoing study. They cite studies that identify adverse events associated with statin therapy but fail to cite systematic reviews that show no increased risk of psychological outcomes, fractures, acute renal failure, arthritis, 
or venous thromboembolism. ${ }^{6-9}$ The incidence of diabetes seems to be real but is linked to the underlying risk of developing diabetes among participants and is also associated with the intensity of statin dose.

Finally, Abramson and colleagues set up a false dichotomy, stating: "Rather than being compelled by guidelines to prescribe statin therapy for people at low risk of cardiovascular disease, doctors would provide a far greater service by explaining the magnitude of the benefits and uncertainty about the harms of statins together with discussion of the epidemiological evidence showing that behavioural risk factors-including tobacco use, lack of physical exercise, and unhealthy diet—are responsible for $80 \%$ of cardiovascular disease." If they (and the BMJ editors) had awaited the publication of the 2013 ACC/AHA cholesterol guidelines, they would have been directed to the companion lifestyle guidelines, which aim to deal with these topics. Salient comment on the importance of considering benefits, harms, and alternative non-drug treatments are embedded in the relevant section of the report. Strong evidence to support the benefits of the type of health promotion proposed by Abramson and colleagues in the primary prevention of cardiovascular disease is sadly lacking.

We have outlined several flaws in Abramson and colleagues "Analysis," which we believe threaten its validity. The decision for patients at low risk of cardiovascular disease to start or continue statins for primary prevention remains under the purview of patients and their doctors. We hope that our Cochrane review, which will continue to be updated as further evidence accrues, will help inform those conversations for better decision making and better health.

Competing interests: $\mathrm{MH}$ receives grant funding from the National Heart, Lung, and Blood Institute and AstraZeneca and travel support from AstraZeneca. SE receives grant funding from the Wellcome Trust and the National Institute of Health Research and royalties from Open University Press.

1 Abramson JD, Rosenberg HG, Jewell N, Wright JM. Should people at low risk of cardiovascular disease take a statin? BMJ 2013;347:f6123. (22 October.)

2 Taylor F, Huffman MD, Macedo AF, Moore TH, Burke M, Davey Smith G, et al. Statins for the primary prevention of cardiovascular disease. Cochrane Database Syst Rev 2013;1:CD004816.

3 Stone NJ, Robinson J, Lichtenstein AH, Bairey Merz CN, Lloyd-Jones DM, Blum CB, et al. $2013 \mathrm{ACC} / \mathrm{AHA}$ guideline on the treatment of blood cholesterol to reduce atherosclerotic cardiovascular risk in adults. J Am Coll Cardiol 2013; published online 7 Nov.

4 Cholesterol Treatment Trialists' Collaborators. The effects of lowering LDL cholesterol with statin therapy in people at low risk of vascular disease: meta-analysis of individual data from 27 randomised trials. Lancet 2012;380:581-90.

5 Kip KE, Hollabaugh K, Marroquin OC, Williams DO. The problem with composite end points in cardiovascular studies. the story of major adverse cardiac events and percutaneous coronary intervention. J Am Coll Cardiol 2008;51:701-7.

6 O'Neil A, Sanna L, Redlich C, Sanderson K, Jacka F, Williams LJ, et al. The impact of statins on psychological wellbeing: a systematic review and meta-analysis. BMC Med 2012;10:154.

7 Toh S, Hernández-Díaz S. Statins and fracture risk. A systematic review. Pharmacoepidemiol Drug Saf 2007;16:627-40.

8 Sandhu S, Wiebe N, Fried LF, Tonelli M. Statins for improving renal outcomes: a meta-analysis. J Am Soc Nephrol 2006;17:2006-16.

9 Rahimi K, Bhala N, Kamphuisen P, Emberson J, Biere-Rafi S, Krane V, et al. Effect of statins on venous thromboembolic events: a meta-analysis of published and unpublished evidence from randomised controlled trials. PLoS Med 2012;9:e1001310.

Cite this as: BMJ 2014;348:g1520

(c) BMJ Publishing Group Ltd 2014 


\section{Table}

Table 1| All cause mortality for low risk patients without previous cardiovascular disease in Cholesterol Treatment Trialists' meta-analysis ${ }^{4}$

\begin{tabular}{lccc}
$\mathbf{5} 5$ year risk of major vascular event & \multicolumn{2}{c}{ No of deaths/No of patients } & Relative risk $(\mathbf{9 5 \%} \mathbf{~ C l})$ \\
\cline { 2 - 3 } & Statin treated & Controls & \\
\hline$<5 \%$ & $129 / 10862$ & $127 / 10993$ & $1.02(0.80$ to 1.30$)$ \\
\hline $5-10 \%$ & $322 / 11344$ & $391 / 11207$ & $0.81(0.70$ to 0.94$)$ \\
\hline Pooled estimate: $\left(I^{2}=57 \%\right)$ & & & $0.86(0.76$ to 0.98$)$ \\
\hline
\end{tabular}

Heterogeneity $\chi^{2}=2.35$ (degrees of freedom 1), $P=0.125$.

Test of pooled relative risk $1, Z=2.32, P=0.02$. 